\title{
Aberrant Driving Behaviours on Risk Involvement among Drivers in China
}

\author{
Hai-peng Shao, Juan Yin (D), Wen-hao Yu, and Qiu-ling Wang \\ College of Transportation Engineering, Chang'an University, Xi'an 710064, China \\ Correspondence should be addressed to Juan Yin; yinj@chd.edu.cn
}

Received 2 April 2020; Revised 18 May 2020; Accepted 4 June 2020; Published 29 June 2020

Academic Editor: Kun Wang

Copyright ( $) 2020$ Hai-peng Shao et al. This is an open access article distributed under the Creative Commons Attribution License, which permits unrestricted use, distribution, and reproduction in any medium, provided the original work is properly cited.

\begin{abstract}
The purpose of this study is to validate the version of Driver Behaviour Questionnaire (DBQ) by considering distractions, fatigue, and drunk driving, the main reasons for accidents in China, as independent parts of violations and errors and further explore the effects of demographic/driving variables and all factors on risk involvement (accident involvement and penalized points). 241 drivers filled in a self-completion questionnaire with 28 items conducted in Xi'an in August 2018. Exploratory factor analysis confirmed a five-factor structure, including violations, distracted driving, errors, drunk driving, and fatigued driving. The frequency of aberrant driving behaviours indicated that distractions were the most prevalent behaviours followed by fatigue. The results showed that drivers with lower education and longer annual mileages were positive with accident involvement while there was no significance in penalized points. Violations and distractions were important factors causing both accidents and penalized points. Therefore, it is effective to reduce accident involvement by establishing educational training and related laws or installing intelligent monitor vehicle equipment to warn drivers to improve safety.
\end{abstract}

\section{Introduction}

With the rapid improvement of living standards, people's demand for private cars is gradually increasing, which also leads to many accidents involving property damage, injuries, and deaths. The China Statistical Yearbook reported that 166,906 car accidents occurred in 2018, killing 46,161 people. The main reasons for accidents include speeding, fatigue, distraction, and drunk driving. And human errors lead to almost $90 \%$ of the accidents [1]. Driving skills and behaviours greatly influence the likelihood of a person exposed to accidents [2]. In general, driving skills are related to driving experience while driving behaviours are related to factors such as a driver's gender, age, habits, personality, and trafficdriving environment [3-5].

To explore the aberrant driving behaviours among drivers, Reason et al. [4] presented a set of driving behaviour questionnaire (DBQ) including 50 items for measuring aberrant driving behaviours, which was divided into 3 factors: violations, errors and lapses. Violations refer to deliberate failure to obey traffic rules or honk behaviours toward others, such as "speeding, crossing the red light, risk overtaking, and honking others to go faster". Errors refer to the failure to achieve planned behaviour due to cognitive judgment, while lapses happen mainly because of inattention or memory failure. This questionnaire is stable and effective in exploring aberrant driving behaviours [6] and has been translated, quoted and revised in many countries [7-9], in which the 27 items DBQ by Parker et al. [10] was widely used. In order to provide a better understanding of driver behaviours, Lawton et al. [11] defined violations as ordinary and aggressive violations while Aberg and Rimmö [12] divided errors into dangerous, inattention and inexperienced errors. Moreover, Guého et al. [3] validated the DBQ with a sample of French drivers and measuring positive driving behaviours [13] toward other users into a questionnaire [3]. In general, scholars have done their best to validate and detail the DBQ depending on different cultural characteristics and naturalistic driving behaviours.

However, few studies consider the distracted driving, fatigued driving, and drunk driving as independent parts of violations and errors to validate the structure of aberrant 
driving behaviours. With the rapid development of in-vehicle technology, drivers using cell phones, navigation systems, and the availability of wireless Internet results in shifting more attention to nondriving related tasks. And it is a major contributor to highway crashes, causing up to $25 \%$ of the crashes [14]. Ledesma et al. [15] developed the Attention-Related Driving Error Scale (ARDES) to record the frequency of inattention and evaluate the predisposition to inattention errors. But the items were about the consequences of distraction (e.g., not notice traffic lights) not the potential sources, such as calling someone or read messages, which directly referred to drivers' distraction. Fatigued driving is another main reason for accidents in many countries $[16,17]$. It results in at least 100,000 accidents in the US each year [18] and accounts for $20 \%$ of the total number of traffic accidents in China [19]. However, the questionnaires about fatigue mainly refer to professional drivers with longer work days or driving hours [20-22]. The items describing drivers' subjective fatigue status in the questionnaire are very less. Besides, a World Health Organization survey showed that $50 \%$ to $60 \%$ of traffic accidents caused by human factors are related to alcohol drinking [23]. The alcohol consumption affects the rate of occurrence of fatal accidents [24]. Therefore, it is significant to add items including distractions, fatigue, and drunk driving to validate the traditional DBQ and explore the relationship between these factors with risk involvement.

There are some researches that explore the demographic/ driving variables associated with aberrant driving behaviours. The results have indicated that males are prone to violations and errors, while females tend to lapses $[4,25,26]$. The age and annual mileages have also entered models and have a validated correlation with violations and errors. Drivers who are young or have more driving exposure are prone to violations and errors $[4,6]$. Furthermore, the driver's self-evaluation, attitude toward laws, and personality are also closely related to driving behaviours $[5,27,28]$.

The relationship between the demographic/driving variables, aberrant driving behaviour, and risk involvement is also worthy exploring. Most studies have indicated that violations [4, 20,29], as well as lapses and errors [30], are significant factors that resulted in accidents. To understand how the demographic/driving variables affected risk involvement, scholars further entered gender, age, annual mileages, educational background, and marital status into a model. Some researches indicated that these variables are not correlated with accidents [31, 32], while some showed that drivers who are young and with long annual mileages had a higher risk of crashes happening [3,33]. Further, the study by Shinar et al. [34] and Vahedi et al. [20] has shown that single drivers declare more accidents.

Therefore, the purpose of this study is (1) to improve the structure of questionnaire considering distracted driving, fatigued driving, and drunk driving as independent parts from violations and errors; (2) to explore the relationship between demographic/driving variables and aberrant driving behaviours; (3) to have a good understanding of both aberrant driving behaviours and risk involvement (accident and penalized points involvement).

\section{Materials and Methods}

2.1. Participants. The data collection was carried out with a cross-sectional survey using a self-completion questionnaire in Xi'an in August, 2018. And the questionnaire distribution was mainly made by the graduate students in the parking lot near larger shopping malls, tourist attractions, and stations. Before recording the data of drivers, a pilot survey was used to test whether the design of the questionnaire is easy to understand among 60 drivers, which resulted in some minor corrections in the items of aberrant driving behaviours. The aim of this survey was apparent to drivers and encouraged them to accurately report his or her demographic/driving variables, risk involvement experience, and the frequency of aberrant driving behaviours. At last, a sample of 300 drivers with validated driving licenses were collected by the face-toface interviews, and 59 questionnaires were excluded from the questionnaires because of incomplete and obvious errors. Therefore, a total of 241 drivers (193 male, 48 female) were included in the analysis. Table 1 shows the distribution of drivers' demographic/driving characteristics and risk involvement. Most drivers' age was between 31 and 45 years, and the average age is 36.60 years $(\mathrm{SD}=7.38)$. The reported statistics showed that drivers had a mean of 9.71 years' driving experience $(S D=5.9)$ with a mean of 33,200 annual mileages $(S D=50,700)$. There was a significant difference from statistics between male and female drivers from the demographic distribution. The sample of male drivers declared more driving experience and annual mileages. Females scored their driving skills as more ordinary or poor while males considered it was excellent. Other characteristics including education, marital status, and risk involvement showed no significant difference.

2.2. Materials. Based on the mature DBQ [4], a new improved version of DBQ considering the main cause of accidents was produced with 28 items, including violations, distracted driving, errors, drunk driving, and fatigued driving. Violation was defined as a deliberate deviation from the legal rules or conflict with other drivers, which contained 10 items referring to the ordinary and aggressive behaviours including speeding, running red lights, frequent changing lanes, and walking through the zebra before pedestrian (e.g., "Speeding when not monitored; Honk to the driver in front to go faster or give way"). Six items referred to errors, which were cognitive or inexperienced errors (e.g., "Failure to judge the speed and distance of an oncoming car when overtaking; Fail to check the rear/side mirror before lane changing"). Diversion of a driver's attention could either be from sources internal or external to the vehicle. This part included 5 items describing the distracted behaviours because of internal person or intelligent equipment, including talking with passengers, calling for someone, and operating in-vehicle systems. Three items were corresponding to the driver's frequency in falling fatigue (e.g., "Frequently fell sleepy because of insufficient sleep time or higher work pressure when driving; Suddenly wake up after driving for a long time on the highway"), and the items mainly described 
TABLE 1: Distribution of driver' demographic/driving variables.

\begin{tabular}{|c|c|}
\hline & Number \\
\hline \multicolumn{2}{|l|}{ Demographic variable } \\
\hline \multicolumn{2}{|l|}{ Age } \\
\hline $22-30$ & 64 \\
\hline $31-45$ & 150 \\
\hline $46-56$ & 27 \\
\hline \multicolumn{2}{|l|}{ Marital status } \\
\hline Single/divorced & 37 \\
\hline Married & 204 \\
\hline \multicolumn{2}{|l|}{ Educational background } \\
\hline Higher than high school & 204 \\
\hline Other & 37 \\
\hline \multicolumn{2}{|l|}{ Driving experience } \\
\hline$\leq 3$ & 38 \\
\hline $4-6$ & 46 \\
\hline $7-10$ & 84 \\
\hline$>10$ & 73 \\
\hline \multicolumn{2}{|l|}{ Driving variable } \\
\hline \multicolumn{2}{|l|}{ Average annual mileage } \\
\hline$\leq 10,000$ & 81 \\
\hline $10,000-20,000$ & 76 \\
\hline $20,000-50,000$ & 51 \\
\hline$>50,000$ & 33 \\
\hline \multicolumn{2}{|l|}{ Scores of driving skills } \\
\hline Excellent & 60 \\
\hline Ordinary/poor & 181 \\
\hline \multicolumn{2}{|c|}{ Accidents during recent 3 years } \\
\hline Yes & 116 \\
\hline No & 125 \\
\hline \multicolumn{2}{|c|}{ Penalized points during recent 1 year } \\
\hline Yes ( $>2$ points) & 116 \\
\hline No ( $\leq 2$ points) & 125 \\
\hline
\end{tabular}

the fatigue fact because of sleep deprivation, extensive driving, and monotonous driving environment. The remaining items were defined as drunk driving, and the items were mainly referred to driver's attitude toward drunk driving after drinking (e.g., "Drive after having a little of alcohol when there are no policemen; Drive back even though you may be over the legal blood-alcohol limit"). For each item, drivers were asked to choose one scale of the frequency about aberrant behaviours described, and the fivepoint scale was ranging from " $1=$ never" to " $5=$ nearly all the time".

Additionally, the information of drivers' gender, age, marital status, educational background, driving experience, annual mileages, and score of driving skills is recorded. To have a great understanding of the relationship between aberrant behaviours and risk involvement, the receiving penalized points over the past year and accident history over the past 3 years were also gathered.

2.3. Methods. To reveal the overall scores of the DBQ items, descriptive statistics were applied. Principal component analysis (PCA) with varimax rotation and iteration was used to test the dimensional structure of the DBQ. The scree plot and Kaiser criterion (an eigenvalue above 1.00 was considered to be a significant value) was used to determine the number of extracted dimensions. And a factor loading above 0.40 was used as a criterion for items to be retained in the DBQ components [20,35]. To test the internal consistency and reliability of the scales, Cronbach's (alpha) was calculated.

Multiple regression models were proposed to study the demographic/driving variables associated with aberrant driving behaviours and two binary logistic models were used to evaluate the relationship between demographic/driving variables, aberrant behaviours, and risk involvement (accidents and penalized points). Moreover, the dichotomous variable (yes/no) about accident involvement (with $0=$ no crash in recent 3 years and $1=$ at least one crash) and penalized points (with $0=$ penalized points less than or equal to 2 points at last year and $1=$ penalized points more than 2 points) was defined as the dependent variable in the binary logistic models. The variables were found statistically significant in a $95 \%$ confidence interval. These statistical analyses were carried out in SPSS 26.0.

\section{Results and Discussion}

\subsection{Results}

3.1.1. Items of Driver Behaviours Questionnaire. Table 2 shows the means and standard deviations for each item in the improved DBQ. The most frequent behaviour was talking with passengers and other items of distraction, which indicated that distractions were more serious aberrant behaviours. This was followed by fatigue behaviours. On the violation item, "see the headlights coming at night, turn the headlights too" was highly scored, which showed the aggressive behaviours were heavier. Speeding as a frequent behaviour had a mean of $1.77(\mathrm{SD}=0.88)$, which also had a higher score. The item, "fail to notice give-way signs for pedestrians and cyclists," was the highest reported error. As expected, the frequency of drunk driving was the lowest because of serious legal punishment.

3.1.2. Principal Component Analysis (PCA) of Driver Behaviour Questionnaire. In order to explore the dimensional structure of the improved DBQ, the PCA with a varimax rotation and iteration was applied. To determine suitability for component analysis, the Kaiser-Meyer-Olkin Test and Bartlett's test were conducted and the results were satisfying $(\mathrm{KMO}=0.862, P$ value $<0.001)$. The eigenvalues of seven factors were above 1 and a cut-off point of 0.4 was used for item loading values. The results showed that factor 3 and factor 4 were all about distraction while factor 6 and factor 7 were corresponding to errors, so factors 3 and 4 were combined into one factor, distracted driving, and factors 6 and 7 were combined into errors. At last, a five-factor structure solution accounted for $56.45 \%$ of the total variance. Cronbach' $\alpha$ alphas had been calculated for each scale and the results ranged from 0.622 to 0.860 . All the results are presented in Table 2.

The first factor, "violations," explained $26.07 \%$ of the variance. It included 10 items: 4 items were aggressive violations and other items were ordinary violations. The 
TABLE 2: Five-factor solution (varimax rotation) for the Driver Behaviour Questionnaire.

\begin{tabular}{|c|c|c|c|c|c|c|}
\hline \multirow{2}{*}{ Structure } & \multirow{2}{*}{$\mathrm{M}(\mathrm{SD})$} & \multicolumn{5}{|c|}{ Loadings } \\
\hline & & 1 & 2 & 3 & 4 & 5 \\
\hline \multicolumn{7}{|l|}{ 1. Violations (Cronbach's $\alpha=0.860$ ) } \\
\hline (Q23) Disregard the speed limits late at night or very early in the morning & $\begin{array}{c}1.95 \\
(0.99)\end{array}$ & 0.70 & & & & \\
\hline (Q11) Speeding through the intersection at the end of the yellow light & $\begin{array}{l}1.63 \\
(0.83)\end{array}$ & 0.67 & & & & \\
\hline (Q8) Honk to the driver in front to go faster or give way & $\begin{array}{c}1.88 \\
(0.87)\end{array}$ & 0.65 & & & & \\
\hline (Q21) Speeding when not monitored & $\begin{array}{l}1.77 \\
(0.88)\end{array}$ & 0.63 & & & & \\
\hline (Q27) Keep close distance with the front car & $\begin{array}{c}1.52 \\
(0.71)\end{array}$ & 0.62 & & & & \\
\hline (Q10) When seeing the headlights coming on at night, turn on the headlights too & $\begin{array}{c}2.02 \\
(1.05)\end{array}$ & 0.61 & & & & \\
\hline (Q4) Continuous lane change or rapid acceleration/deceleration & $\begin{array}{l}1.58 \\
(0.79)\end{array}$ & 0.61 & & & & \\
\hline (Q15) Pass a slow driver on the right side & $\begin{array}{l}1.56 \\
(0.78)\end{array}$ & 0.59 & & & & \\
\hline (Q7) Crossing red-light signal when not monitored & $\begin{array}{c}1.55 \\
(0.78)\end{array}$ & 0.57 & & & & \\
\hline (Q19) Try to walk through the zebra crossing firstly before pedestrian. & $\begin{array}{c}1.42 \\
(0.73)\end{array}$ & 0.52 & & & & \\
\hline
\end{tabular}

\section{Distracted driving (Cronbach's $\alpha=0.666)$}

(Q12) Operate the on-board system while driving (such as playing music, adjusting radio, or air- 2.41 conditioning)

(Q22) Talk with a passenger when driving

(Q1) Call someone when driving

(Q16) Visit the web or watch movies while driving

(Q25) Read messages while driving (SMS, WeChat, or QQ messages)

3. Errors (Cronbach's $\alpha=0.649$ )

(Q2) Fail to check the rear/side mirror before lane changing

(Q3) Fail to judge the speed and distance of an oncoming car when overtaking

$1.6(0.64)$

1.44

$(0.66)$

2.01

(1.09)

1.55

(0.86)

1.73

$(0.82)$

1.76

(1.22)

(Q13) Brake suddenly on the slippery road.

4. Drunk driving (Cronbach's $\alpha=0.622$ )

(Q20) Drive after a little of alcohol when there are no policemen

$1.1(0.34)$

1.13

$(0.37)$

(Q14) Drive after a little of alcohol when needing to take to someone else home

1.15

$(0.46)$

1.15

$(0.48)$

0.59

(Q17) Drive back even though you may be over the legal blood-alcohol limit

5. Fatigued driving (Cronbach's $\alpha=0.638$ )

(Q18) Feel tired or sleepy when driving

0.61

(Q24) Frequently fell sleepy because of insufficient sleep time or higher work pressure when driving

${ }^{1} \mathrm{~A}$ cut-off point of 0.40 was used for item loading values and factor loadings below 0.40 were omitted for the sake of clarity. 
second factor, "distracted driving," explained $9.40 \%$ of the variance. All items tended to record the frequency of distraction because of calling someone or other in-vehicle equipment. The third factor, "errors," explained $8.02 \%$ of the variance. It included 6 items, which were mainly about cognitive and inexperienced errors. The fourth factor, "drunk driving," explained $7.38 \%$ of the variance with items committing to driver's attitude after drinking alcohol. And the last factor, "fatigued driving," explained $5.58 \%$ of the variance. All items corresponded to fatigue because of sleep problems, work pressure, and boring driving situations.

3.1.3. Variables Associated with Five-Factor Scores. To explore the relationship between demographic/driving variables and aberrant driving behaviours, five multiple regression models were established. Standardized regression coefficients and significance levels are shown in Table 3. The explanatory variables included the dichotomous variables (gender, marital status, educational background, and scores of driving skills) and the continuous variables (age, driving experience, and annual mileages). And the values of the explanatory variables are shown in Table 4 .

Violations were associated with gender, age, and scores of driving skills. Male drivers who were young and confident in their driving skills declared more violations. These variables accounted for $6.0 \%$ of the variance in the violation scores. All the variables were not significant for drunk driving. The reported distracted driving was predicted by drivers' age and scores of driving skills. Drivers who were young and confident in their driving skills were more probable to take part in nondriving related tasks, and these variables accounted for $5.0 \%$ of the variance in the distraction scores. The reported errors were associated with gender and age. The young males were significantly prone to make mistakes. Both variables accounted for $4.0 \%$ of the variance in the errors scores. Fatigued driving was also associated with males, which accounted for $4.0 \%$ of the variance in the fatigue scores. The marital status, driving experience, and annual mileages were not significant to the aberrant driving behaviours.

3.1.4. Variables Associated with Risk Involvement. To explore the relationship between demographic/driving variables, aberrant driving behaviours and risk involvement, two binary logistic regression models were performed. Crashes and penalized points are dependent variables, respectively, with demographic/driving variables and five DBQ scores as explanatory variables. The results are shown in Table 5. The first model of accident involvement was reliable (chisquare $=62.26, \mathrm{df}=12, P<0.001, P=0.683$ to the Hosmer-Lemeshow test) and the second too (chi-square $=25.26$, $\mathrm{df}=12, P<0.05, P=0.935$ to the Hosmer-Lemeshow test). Overall, $69.3 \%$ of the predictions were accurate for both models. The first model successfully predicted $76.8 \%$ of drivers without an accident and $61.2 \%$ with at least one crash. And the second model successfully predicted $91.0 \%$ of drivers without an accident and only $29.4 \%$ with at least one crash.
The first model indicated that drivers who had a lower educational background and longer annual mileages declared more crashes while the second model did not find significantly demographic/driving variables associated with penalized points. Violations and distractions were positively related to both accident involvement and penalized points. The errors, fatigue, and drunk driving were not significant to both dependent variables.

3.2. Discussion. The aim of this study is to validate the structure of DBQ with a sample of Chinese drivers and precisely explore the relationship between demographic/ driving variables, aberrant driving behaviours, and risk involvement.

3.2.1. Validating the Structure of $D B Q$. The previous researches mainly divided the aberrant driving behaviours into 3 parts: violations, errors, slips, and lapses [4]. Lawton et al. [11] divided violations into ordinary and aggressive violations. The errors were also differentiated into inexperience, inattention, and dangerous errors [3]. The items of fatigue and drunk driving are included in violation, while distractions are included in errors or inattention errors. To precisely understand the aberrant driving behaviours, the fatigued driving and drunk driving were separated from violations as two independent parts as well as distracted driving from errors.

The new DBQ consisting of 28 items, and a PCA produced a five-factor structure. The reliability and validity of the questionnaire indicated that the factor structure was credible. The items of violations and errors are mainly produced depending on reason's DBQ [4]. There were also some items added because of complex motor and nonmotor mixed traffic or vehicles not giving way to pedestrians in China [21]. The designed items of distraction mainly tended to drivers' attention shifting from driving tasks to in-vehicle equipment, which had a difference with inattention errors referring to the driving consequence. The results of the questionnaire indicated that distraction was the most serious and frequent behaviour in naturalistic driving, especially in operating in-vehicle equipment or calling someone. This discovery is in line with researches in the United States by Schroeder [36] who found that drivers engage in while driving include talking with other passengers, adjusting the car radio, making and receiving cell phone calls, and using a smartphone for driving directions. The items designed for fatigue mainly pointed to drivers who did not have good sleep habits or had higher work pressure and a monotonous driving environment. The answers to these items indicated drivers' attitude and daily status toward fatigue. And the results showed that fatigued driving was another frequent behaviour. The items of drunk driving were prone to drivers' moral norm and driving attitude. The score of this factor was lower among other factors, which indicated that the penalty of drunk driving had a great impact on traffic safety. In general, the new DBQ enriches the conventional structure by adding the significant reasons of accidents in China. 
TABLE 3: Standardized $\beta$ of multiple linear regression with five factors as the dependent variable.

\begin{tabular}{|c|c|c|c|c|c|}
\hline & Violations & Distracted driving & Errors & Drunk driving & Fatigued driving \\
\hline Gender & $-0.18^{*}$ & -0.03 & $-0.14^{*}$ & -0.09 & $-0.23^{*}$ \\
\hline Age & $-0.19^{*}$ & $-0.16^{*}$ & $-0.20^{*}$ & 0.06 & -0.08 \\
\hline Marital status & 0.05 & -0.02 & 0.07 & -0.03 & -0.05 \\
\hline Educational background & 0.02 & 0.08 & -0.03 & -0.09 & 0.02 \\
\hline Driving experience & -0.03 & -0.05 & -0.06 & -0.10 & 0.12 \\
\hline Average annual mileage & -0.01 & -0.05 & 0.04 & 0.03 & -0.04 \\
\hline Scores of driving skills & $-0.16^{*}$ & $-0.16^{*}$ & -0.06 & -0.08 & 0.08 \\
\hline$R^{2}$ & 0.09 & 0.08 & 0.06 & 0.03 & 0.07 \\
\hline Adjusted $R^{2}$ & 0.06 & 0.05 & 0.04 & -0.01 & 0.04 \\
\hline
\end{tabular}

TABLE 4: The values of the explanatory variables.

\begin{tabular}{lc}
\hline Characteristic & Values \\
\hline Gender & Male $=0$, female $=1$ \\
Age & Continuous variables \\
Marital status & Single/divorced $=0$, married $=1$ \\
Educational background & Higher than high school $=1$, other $=0$ \\
Driving experience & Continuous variables \\
Average annual mileage & Continuous variables \\
Scores of driving skills & Excellent $=0$, ordinary $/$ poor $=1$ \\
\hline
\end{tabular}

TABLE 5: Variables associated with risk involvement.

\begin{tabular}{lcccccc}
\hline & \multicolumn{3}{c}{ Accident } & \multicolumn{3}{c}{ Penalized points } \\
Variables & involvement & \multicolumn{3}{c}{ involvement } \\
& $\mathrm{B}$ & OR & Sig & $\mathrm{B}$ & OR & Sig \\
\hline Gender & -0.04 & 0.96 & 0.91 & 0.52 & 1.68 & 0.18 \\
Age & -0.01 & 0.99 & 0.87 & -0.03 & 0.98 & 0.39 \\
Marital status & 0.23 & 1.26 & 0.62 & 0.07 & 1.07 & 0.89 \\
Educational background & $-1.04^{*}$ & 0.35 & 0.02 & -0.17 & 0.84 & 0.69 \\
Driving experience & -0.02 & 0.98 & 0.64 & -0.01 & 0.99 & 0.75 \\
Average annual mileage & $0.08^{*}$ & 1.09 & 0.02 & 0.05 & 1.05 & 0.09 \\
Scores of driving skills & -0.61 & 0.54 & 0.11 & -0.15 & 0.86 & 0.68 \\
Violations & $0.89^{*}$ & 2.44 & 0.03 & $1.14^{*}$ & 3.13 & 0.01 \\
Distracted driving & $1.04^{* *}$ & 2.84 & 0.00 & $0.80^{*}$ & 2.22 & 0.02 \\
Errors & 0.09 & 1.09 & 0.81 & 0.01 & 1.01 & 0.97 \\
Drunk driving & 0.55 & 1.73 & 0.37 & 0.45 & 1.57 & 0.44 \\
Fatigued driving & 0.14 & 1.15 & 0.56 & 0.21 & 1.23 & 0.39 \\
\hline
\end{tabular}

${ }^{1 *} P<0.05,{ }^{* *} P<0.01$. B: regression coefficient of variables in the model. OR: odds ratio, the odds of a dependent variable increase $(\mathrm{OR}>1)$ or decrease $(\mathrm{OR}<1)$ when the value of the explanatory variable increases by 1.0 unit. Sig: significance of explanatory variables in the model.

\subsubsection{Relationship between Demographic/Driving Variables,} Aberrant Driving Behaviours, and Risk Involvement. The results showed that male drivers declared more violations than the female drivers, which was in line with many other studies $[3,4,25,26]$. There was also a gender difference in errors that male drivers declared more errors, such as failure to notice signs or pedestrians, which was in line with the literature in which males report more violations, errors, and lapses [33]. The results also indicated that males declared more fatigue, which was consistent with the previous study [37]. The results may be because most sample is male drivers who have longer mileages. It was expected that the results did not find a gender difference in drunk driving, which can explain that the traffic laws forbidding drunk driving had significant meaning in curbing driving after drinking.

The findings that young drivers declared more violations were keeping consistent with previous literatures [3, 4]. The errors were also associated with age, which was in line with the previous studies that errors dropped with age $[6,31]$ not the different opinion that errors increased with age [20,25]. The elder drivers had access to more driving experience, so the errors happening were lower than the young. The items of distracted driving mainly included distraction due to invehicle intelligent equipment, so the young had a higher inclination to distraction. However, the results did not find an association between age and fatigue or drunk driving. The reason may be that the age range of participates was mainly in 31-45 years old and driving hours were less every day. Therefore, it is not easier to feel fatigue compared to professional drivers. With the more serious traffic laws in forbidding drunk driving, all of drivers had tried their best to obey the laws, so there was no relationship found between age and drunk driving. Furthermore, the drivers who were confident with their driving skills were prone to violation and distraction, which is in line with the results of Reason et al. [4] that drivers who considered themselves with a high level of driving skills tend to more violations. With selfconfident driving skills, drivers may relax and shift more attention from roads to in-vehicle equipment, which makes drivers inattentive and add the chance to violation. However, drivers' marital status, driving experience, and annual mileage did not have a significant correlation with the five factors.

The results showed that drivers with lower educational background and longer annual mileages declared more accidents, which was in line with the literatures that drivers with longer mileage had high propensity to crashes $[3,6,20]$. With longer annual mileages, the chance of drivers exposed to the road is larger, so it is easier for accidents to happen. However, the study by Vahedi et al. [20] found no significance between educational background and accidents. Other demographic/driving variables did not find a significant correlation with the accident, which was not in line with the studies that single [20,34] and young drivers $[3,4,10]$ were more likely involved in the accident. Besides, no correlation between demographic/driving variables and penalized points was found. 
The findings showed that violations were the key factor attributed to the accident, which is in line with the previous studies $[3,4,6,10,29,31]$. And the study also found that both violations and distracted driving were positively correlated with accident involvement and penalized points. However, the results did not find a positive correlation between fatigue and risk involvement, which was not in line with the study by Wang et al. [22] that found fatigue was positively related to crash involvement. The reason may be that the samples were unprofessional drivers with less driving hours and workload than the professional. No significance between drunk driving and risk involvement was found. This may be explained by more serious legal punishment in drunk driving. Therefore, it is important to propose more traffic laws and fines to improve traffic safety [21].

\section{Conclusions}

Most previous researches have divided the DBQ into 3-5factor structure, including ordinary/aggressive violation and inattention/inexperienced/dangerous errors. Little researches corresponded to the cause of accidents to subdivide the structure, so the purpose of this study is to validate the DBQ by divided distractions, fatigue, and drunk driving from violations and errors. Furthermore, the relationship between demographic/driving variables and aberrant driving behaviours was explored. The results showed that male drivers declared more violations, errors, and fatigued driving. And young drivers tended to more violations, distractions, and errors. Drivers who were confident in their driving skills easily led to violations and distractions. The relationship between demographic/driving variables, aberrant driving behaviours, and risk involvement indicated that drivers with lower education and higher annual mileage declared more accidents. And both violations and distracted driving were positively correlated with accident involvement and penalized points. Therefore, the findings of this article could be used to intervene in the driver's aberrant behaviours. it is effective to establish educational training or related laws or installing intelligent vehicle monitor equipment to avoid driver's violations and distractions to reduce risk involvement.

\section{Limitation}

The limitation of this study mainly includes the following. (1) The frequency of aberrant driving behaviours is recorded by self-completion questionnaires, so drivers may be affected by sociopsychological expectations and response dishonestly, which will affect subsequent analysis. (2) The sample is not large and balanced in demographic/driving variables and does not cover other cities. (3) Furthermore, the stability of the DBQ questionnaire structure can be affected by different DBQ items, driving environments, and cultures. Therefore, the applicability of this structure should be tested in different countries. In general, the results of the questionnaire have a significant correlation with drivers' naturalistic driving behaviours, so its structure can objectively measure aberrant driving behaviours. To further study the relationship between aberrant driving behaviours and risk involvement, the data from the real driving experiments as in a previous study [38] is also necessary to be recorded.

\section{Data Availability}

The data of participates responding to the DBQ are available from the corresponding author (yinj@chd.edu.cn).

\section{Conflicts of Interest}

The authors declare that there are no conflicts of interest regarding the publication of this paper.

\section{Acknowledgments}

This research was supported by the National Key R\&D Program of China (2019YFB1600302), the Fundamental Research Funds for the Central Universities (CHD: 300102219210 and CHD: 300102210201 ), and the 111 Project of Sustainable Transportation for Urban Agglomeration in Western China (No. B20035). The authors would like to thank the participants for their cooperation.

\section{References}

[1] A. Bener and R. Alwash, "A perspective on motor vehicle crash injuries and speeding in the United Arab Emirates," Traffic Injury Prevention, vol. 3, no. 1, pp. 61-64, 2002.

[2] H. A. Deery and A. W. Love, "The effect of a moderate dose of alcohol on the traffic hazard perception profile of young drink-drivers," Addiction, vol. 91, no. 6, pp. 815-828, 1996.

[3] L. Guého, M.-A. Granié, and J.-C. Abric, "French validation of a new version of the driver behavior questionnaire (DBQ) for drivers of all ages and level of experiences," Accident Analysis \& Prevention, vol. 63, pp. 41-48, 2014.

[4] J. Reason, A. Manstead, S. Stradling, J. Baxter, and K. Campbell, "Errors and violations on the roads: a real distinction?" Ergonomics, vol. 33, no. 10-11, pp. 1315-1332, 1990.

[5] J. Yang, F. Du, W. Qu, Z. Gong, and X. Sun, "Effects of personality on risky driving behavior and accident involvement for chinese drivers," Traffic Injury Prevention, vol. 14, no. 6, pp. 565-571, 2013.

[6] J. C. F.D. Winter and D. Dodou, "The driver behaviour questionnaire as a predictor of accidents: a meta-analysis," Journal of Safety Research, vol. 41, no. 6, pp. 463-470, 2010.

[7] P. N. Blockey and L. R. Hartley, "Aberrant driving behaviour: errors and violations," Ergonomics, vol. 38, no. 9, pp. 17591771, 1995.

[8] C. Gabaude, J.-C. Marquié, and F. Obriot-Claudel, "Selfregulatory driving behaviour in the elderly: relationships with aberrant driving behaviours and perceived abilities," Le Travail Humain, vol. 73, no. 1, pp. 31-52, 2010.

[9] M. J. Sullman, M. Meadows, and K. Pajo, "Errors, lapses and violations in the drivers of heavy vehicles," in Proceedings of International Conference on Traffic and Transport PsychologyICTTP 2000, Held 4-7 September 2000, Berne, SwitzerlandKeynotes, Symposia, Thematic Sessions, Workshops, Posters, List of Participants and Word Viewer-Cd Rom, Bern, Switzerland, September 2004. 
[10] D. Parker, J. T. Reason, A. S. R. Manstead, and S. G. Stradling, "Driving errors, driving violations and accident involvement," Ergonomics, vol. 38, no. 5, pp. 1036-1048, 1995.

[11] R. Lawton, D. Parker, A. S. R. Manstead, and S. G. Stradling, "The role of affect in predicting social behaviors: the case of road traffic violations," Journal of Applied Social Psychology, vol. 27, no. 14, pp. 1258-1276, 1997.

[12] L. Aberg and P.-A. Rimmo, "Dimensions of aberrant driver behaviour," Ergonomics, vol. 41, no. 1, pp. 39-56, 1998.

[13] T. Özkan and T. Lajunen, "A new addition to DBQ: positive driver behaviours scale," Transportation Research Part F: Traffic Psychology and Behaviour, vol. 8, no. 4-5, pp. 355-368, 2005.

[14] J. C. Stutts, D. W. Reinfurt, L. Staplin, and E. Rodgman, "The role of driver distraction in traffic crashes," 2001.

[15] R. D. Ledesma, S. A. Montes, F. M. Poó, and M. F. LópezRamón, "Individual differences in driver inattention: the attention-related driving errors scale," Traffic Injury Prevention, vol. 11, no. 2, pp. 142-150, 2010.

[16] A. Bener, E. Yildirim, T. Özkan, and T. Lajunen, "Driver sleepiness, fatigue, careless behavior and risk of motor vehicle crash and injury: population based case and control study," Journal of Traffic and Transportation Engineering (English Edition), vol. 4, no. 5, pp. 496-502, 2017.

[17] X. Wang and C. Xu, "Driver drowsiness detection based on non-intrusive metrics considering individual specifics," Accident Analysis \& Prevention, vol. 95, pp. 350-357, 2016.

[18] C. Liu and R. Subramanian, Factors Related to Fatal SingleVehicle Run-Off-Road Crashes, Createspace Independent Pub, 2013pp. 17-18, Createspace Independent Pub, Scotts Valley, CA, USA, No. HS-811 231.

[19] L. Liu, X. Deng, and X. Ying, "Development of detecting techniques and devices for vehicle driver fatigue," Chinese Medical Equipment Journal, vol. 12, pp. 28-30, 2006.

[20] J. Vahedi, A. Shariat Mohaymany, Z. Tabibi, and M. Mehdizadeh, "Aberrant driving behaviour, risk involvement, and their related factors among taxi drivers," International Journal of Environmental Research and Public Health, vol. 15, no. 8, p. 1626, 2018.

[21] Y. Wang, L. Li, L. Feng, and H. Peng, "Professional drivers' views on risky driving behaviors and accident liability: a questionnaire survey in Xining, China," Transportation Letters, vol. 6, no. 3, pp. 126-135, 2014.

[22] Y. Wang, L. Li, and C. G. Prato, "The relation between working conditions, aberrant driving behaviour and crash propensity among taxi drivers in China," Accident Analysis \& Prevention, vol. 126, pp. 17-24, 2019.

[23] M. Peden, R. Scurfield, D. Sleet et al., World Report on Road Traffic Injury Prevention, pp. 80-83, World Health Organization, Geneva, Switzerland, 2004.

[24] H. L. Chang and C.-C. Yeh, "The life cycle of the policy for preventing road accidents: an empirical example of the policy for reducing drunk driving crashes in Taipei," Accident Analysis \& Prevention, vol. 36, no. 5, pp. 809-818, 2004.

[25] J. Mesken, T. Lajunen, and H. Summala, "Interpersonal violations, speeding violations and their relation to accident involvement in Finland," Ergonomics, vol. 45, no. 7, pp. 469-483, 2002.

[26] J. Shi, Y. Bai, X. Ying, and P. Atchley, "Aberrant driving behaviors: a study of drivers in Beijing," Accident Analysis \& Prevention, vol. 42, no. 4, pp. 1031-1040, 2010.

[27] B. Y. S. Jing, "Driving behaviour and influential factors study in beijing," Journal of Transport Information and Safety, vol. 28, no. 2, pp. 114-119, 2010.
[28] M. Ma, X. Yan, H. Huang, and M. Abdel-Aty, "Safety of public transportation occupational drivers: risk perception, attitudes, and driving behavior," Transportation Research Record: Journal of the Transportation Research Board, vol. 2145, no. 1, pp. 72-79, 2010.

[29] M. J. M. Sullman, M. L. Meadows, and K. B. Pajo, "Aberrant driving behaviours amongst New Zealand truck drivers," Transportation Research Part F: Traffic Psychology and Behaviour, vol. 5, no. 3, pp. 217-232, 2002.

[30] A. Bener, T. Özkan, and T. Lajunen, "The driver behaviour questionnaire in arab gulf countries: Qatar and United Arab Emirates," Accident Analysis \& Prevention, vol. 40, no. 4, pp. 1411-1417, 2008.

[31] M. E. Gras, M. J. Sullman, M. Cunill, M. Planes, M. Aymerich, and S. Font-Mayolas, "Spanish drivers and their aberrant driving behaviours," Transportation Research Part F: Traffic Psychology and Behaviour, vol. 9, no. 2, pp. 129-137, 2006.

[32] T. Nordfjærn, A. M. Hezaveh, and A. R. Mamdoohi, "An analysis of reported driver behaviour in samples of domestic and expatriate Iranians," Journal of Risk Research, vol. 18, no. 5, pp. 566-580, 2015.

[33] P. F. Lourens, J. A. M. M. Vissers, and M. Jessurun, “Annual mileage, driving violations, and accident involvement in relation to drivers' sex, age, and level of education," Accident Analysis \& Prevention, vol. 31, no. 5, pp. 593-597, 1999.

[34] D. Shinar, E. Schechtman, and R. Compton, "Self-reports of safe driving behaviors in relationship to sex, age, education and income in the US adult driving population," Accident Analysis \& Prevention, vol. 33, no. 1, pp. 111-116, 2001.

[35] W. Zhang, X. Zhang, Z. Feng, J. Liu, M. Zhou, and K. Wang, "The fitness-to-drive of shift-work taxi drivers with obstructive sleep apnea: an investigation of self-reported driver behavior and skill," Transportation Research Part F: Traffic Psychology and Behaviour, vol. 59, pp. 545-554, 2018.

[36] P. Schroeder, M. Wilbur, R. Pena, and S. Abt, "National survey on distracted driving attitudes and behaviours-2015; United States," National Highway Traffic Safety Administration, pp. 12-70, 2018.

[37] D.-H. Li, Q. Liu, W. Yuan, and H.-X. Liu, "Relationship between fatigue driving and traffic accident," Journal of Traffic and Transportation Engineering (Xi'an, Shaanxi), vol. 10, pp. 12-70, 2010.

[38] Z. Feng, M. Yang, W. Zhang, Y. Du, and H. Bai, "Effect of longitudinal slope of urban underpass tunnels on drivers' heart rate and speed: a study based on a real vehicle experiment," Tunnelling and Underground Space Technology, vol. 81, pp. 525-533, 2018. 